\section{A patient presented with high fever and bloody pericardial effusion (hemorrhagic pericarditis), with literature review}

\section{Abstract}

We report a case of hemorrhagic pericarditis caused by Mycobacterium tuberculosis infection of the pericardium which is an extremely rare diagnosis. The literature review showed that there were rare cases of tuberculosis causing hemorrhagic pericarditis, but the diagnosis was made either at postmortem or not firmly diagnosed. Our patient was definitely diagnosed as hemorrhagic pericarditis due to $M$. tuberculosis, he was treated and was discharged.

\section{Keywords}

Pericarditis; Hemorrhagic Pericarditis; Mycobacterium Tuberculosis;

Tuberculous Pericarditis
Jamal Wadi Al Ramahi ${ }^{1}$, Nidhal Hamad², Hassan Annab3, Kareem Al Khalidi ${ }^{4}$, Omran Abu Khalaf 5 , Mohammad Obaidat ${ }^{6}$

1 School of Medicine, University of Jordan, Office 11, 3rdFloor. Jordan Hospital Medical Center, Amman. Jordan 11118.

2 Cardiologist, Al-Khalidi Medical Center, Amman, Jordan

3 Pathology Section, Laboratory Department. Jordan Hospital and Medical Center. Amman, Jordan 11118

4 Radiology Department. Al-Khalidi Medical Center, Amman, Jordan.

5 Internal Medicine Department. Jordan Hospital. Amman, Jordan.

6 Pharmacy Department. Al-Khalidi Medical Center, Amman, Jordan.

Contact information:

Prof. Jamal Wadi Al Ramahi, M.D, FIDSA.

”jamalwadimd@yahoo.com.

\title{
Introduction
}

Pericarditis is caused by several factors; idiopathic, infections, neoplasm, autoimmune, radiation, post STEMI (ST-segment elevation myocardial infarction) and noxious substances [1]. However, hemorrhagic pericarditis may be caused by some of the factors but at different rates; $M$. tuberculosis is conceptually placed as a "frequent" cause of hemorrhagic pericarditis, despite its occurrence, it is an uncommon cause even among cases of pericarditis in endemic areas [2]. A study from Rohilkhand region (India), a county known to have high tuberculosis prevalence, 322 patients with pericarditis were enrolled between June 2015 - May 2017, among them, 257 (80\%) of the patients had tuberculous pericarditis and none of them presented with hemorrhagic pericarditis including few patients with HIV and tuberculosis co-infection [3]. In another retrospectives single USA medical center 
in the 1990s, hemorrhagic pericarditis causes were etiologically diverse, the leading causes detected in the 96 patients were: iatrogenic 31\%, Malignancy $26 \%$, Complications of acute myocardial infarction $11 \%$, idiopathic $10 \%$, uremic $7 \%$ and $M$. tuberculosis was rare among the other causes (1 patient) which was diagnosed as a postmortem [4]. In addition, mixed connective tissues disease and infliximab were added to the causes in some reports [5-6]

In the current case, we report a case of hemorrhagic pericarditis in a patient who was initially presented in Sudan with chest pain, prostration, and fever. Workup did not reveal the etiology of his illness, he presented to Al Khalidi hospital for evaluation, and hemorrhagic pericarditis due to $M$. tuberculosis was firmly diagnosed.

\section{The case}

A thirty-four years old Sudanese male patient with no underlying morbid condition. He presented to Al Khalidi Hospital/ Medical center emergency department with the complaints of fever of four weeks duration, it was associated with chest pain, productive cough, abdominal pain, diarrhea, and weight loss. On presentation, he looked sick, sleepy and sweating. He was febrile with temperature $\geq$ $38.2^{\circ} \mathrm{C}$ in his initial admissions days, heart rate 138 beat/min and blood pressure 122/75. He appeared weak, could not walk, and sleepy. His heart sounds were distant with no audible murmur and no lungs crepitations, soft abdomen, and no peripheral signs on extremities.

\section{Laboratory}

HIV 1 and 2 antibodies were negative, hemoglobin $11.0 \mathrm{gm} / \mathrm{dl}, \mathrm{MCV}$ 78, WBC 9600/mm3, platelets 337000/mm³, ALT $45 \mathrm{IU} / \mathrm{L}$, AST $61 \mathrm{IU} / \mathrm{L}$. Serum creatinine $0.56 \mathrm{mg} / \mathrm{dl}$, BUN $17.3 \mathrm{mg} / \mathrm{dl}$, bilirubin $0.97 \mathrm{mg} / \mathrm{dl}$. Electrolytes: $\mathrm{Na}+126 \mathrm{mmol} / \mathrm{L}, \mathrm{K}+3.84$ $\mathrm{mmol} / \mathrm{L}, \mathrm{Ca}++8.53 \mathrm{mg} / \mathrm{dl}, \mathrm{Cl}-92 \mathrm{mmol} / \mathrm{L}, \mathrm{CRP} 136$ $\mathrm{mg} / \mathrm{dl}$ and ESR $90 \mathrm{~mm} / 1 \mathrm{st}$ hour. Cardiac troponin blood level $<0.05 \mathrm{ng} / \mathrm{ml}$ and $0.02 \mathrm{ng} / \mathrm{ml}, \mathrm{CKMB}$ $8.1 \mathrm{U} / \mathrm{L}$, BNP 37.73 pg/ml. Procalcitonin 3.123 ng/ml. Two sets of aerobic and anaerobic blood cultures on admission did not grow bacteria (BacT/Alert, Biomerieux). Pericardial fluid aerobic incubation showed no growth. Plain chest X-ray showed cardiomegaly with water bottle sign. Brain MRI was normal. Electrocardiography showed sinus tachycardia with low voltage. 2D-echocardiography (Figure not available) showed moderate posterior pericardial effusion with pericardial thickening and ejection fracture was $>60 \%$. Chest CT-scan with I.V contrast demonstrated large and circumferential pericardial effusion measuring up to $4.5 \mathrm{~cm}$ in thickness, the effusion is relatively high in density (Hounsfield unit of 25) with some septations, the pericardium is thickened measuring $7 \mathrm{~mm}$ in thickness (Figure 1). No pulmonary nodules, small sized multiple mediastinal lymph nodes were noted. Ultrasound-guided pericardiocentesis revealed blood with a thick deep

Figure 1: The pericardium is thickened measuring $7 \mathrm{~mm}$ in thickness, with large and circumferential pericardial effusion measuring up to $4.5 \mathrm{~cm}$ in thickness. The effusion is relatively high in density (Hounsfield unit of 25) and it has some septations.

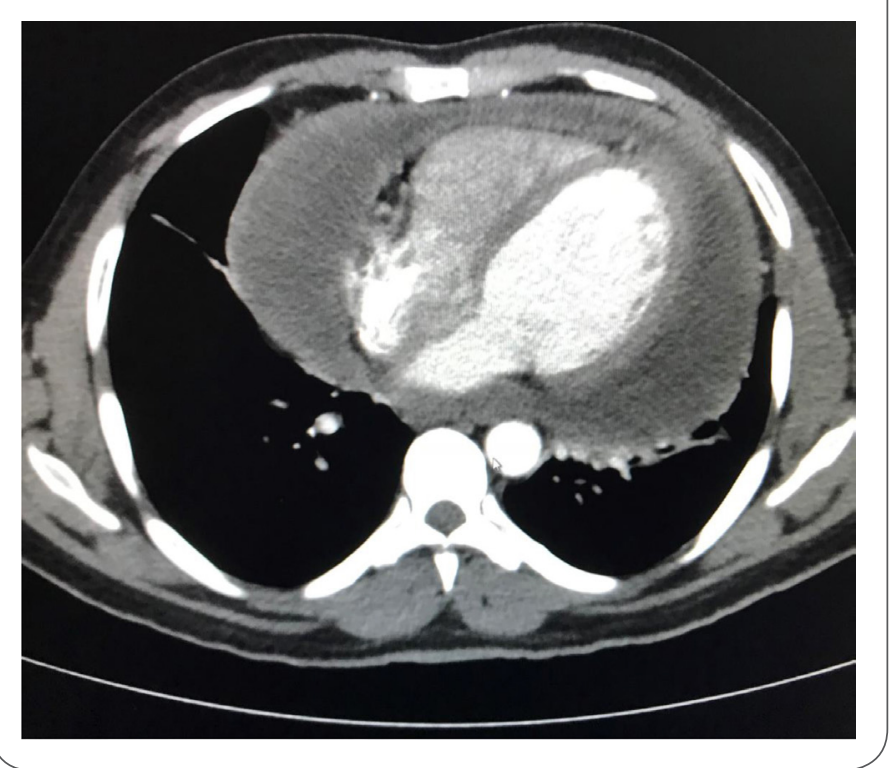


dark color, $200 \mathrm{ml}$ of bloody pericardial fluid was aspirated on 1/11/2018. In the next few days, he remained febrile, though his general condition improved and became more wakeful. The aspirate did not show acid-fast bacteria, malignant cells or bacteria on gram stain and culture. Pericardial fluid samples sent for cytology and analysis showed WBC +2 , no bacteria seen on gram stain, and AFB stain was negative. Empirically, he was treated as a septic patient with hemorrhagic pericarditis with parenteral cefazoline 2 gram every eight hours.

Later, a pericardial window was done, and tissue pathology showed caseating granulomas (Figure 2 A, B) and positive AFB on Ziehl-Neelsen stain (Figure 3). Mycobacterial PCR (GenXpert, Cephid) was posi-

\section{Figure 2.}

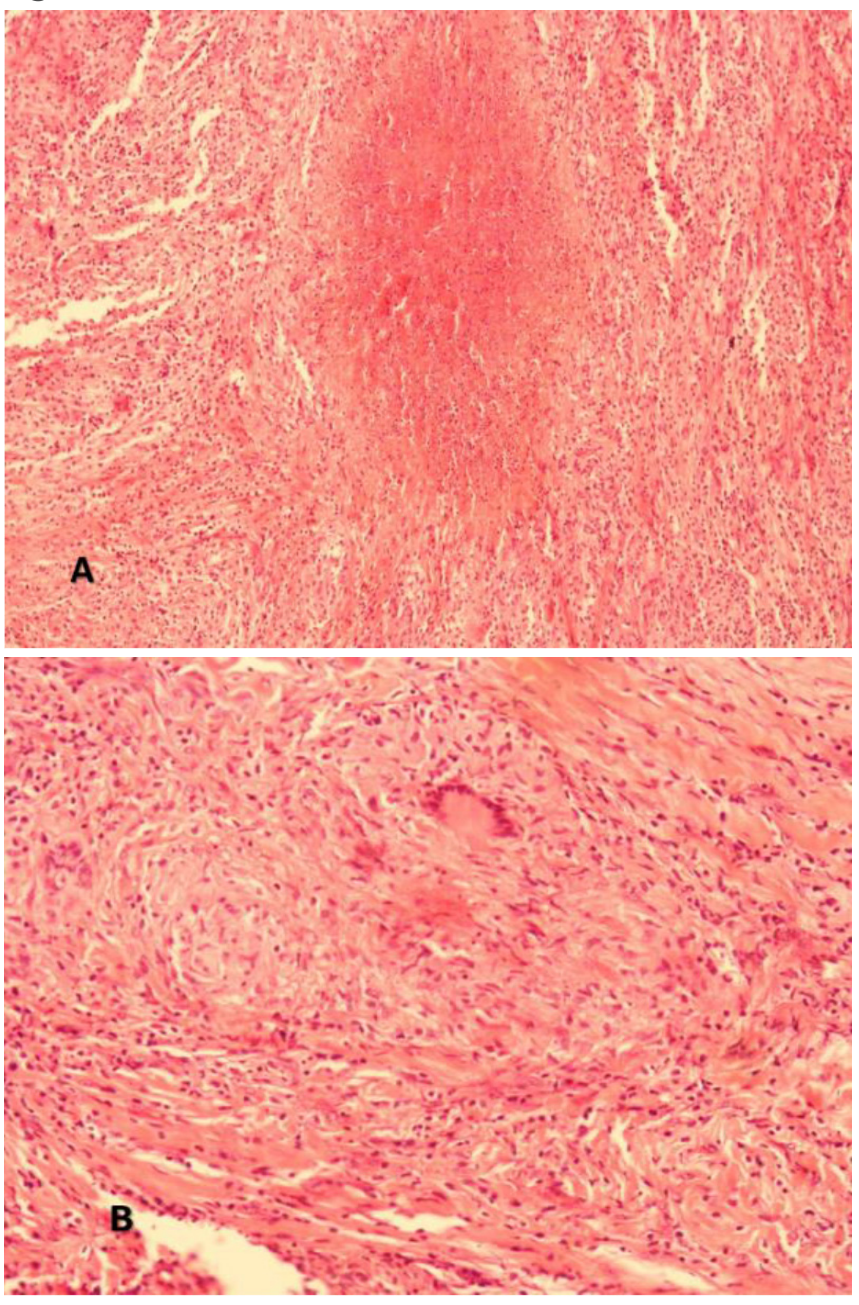

A: shows a granuloma with caseation necrosis; B) shows the caseation with Langerhans multinucleated giant cells.

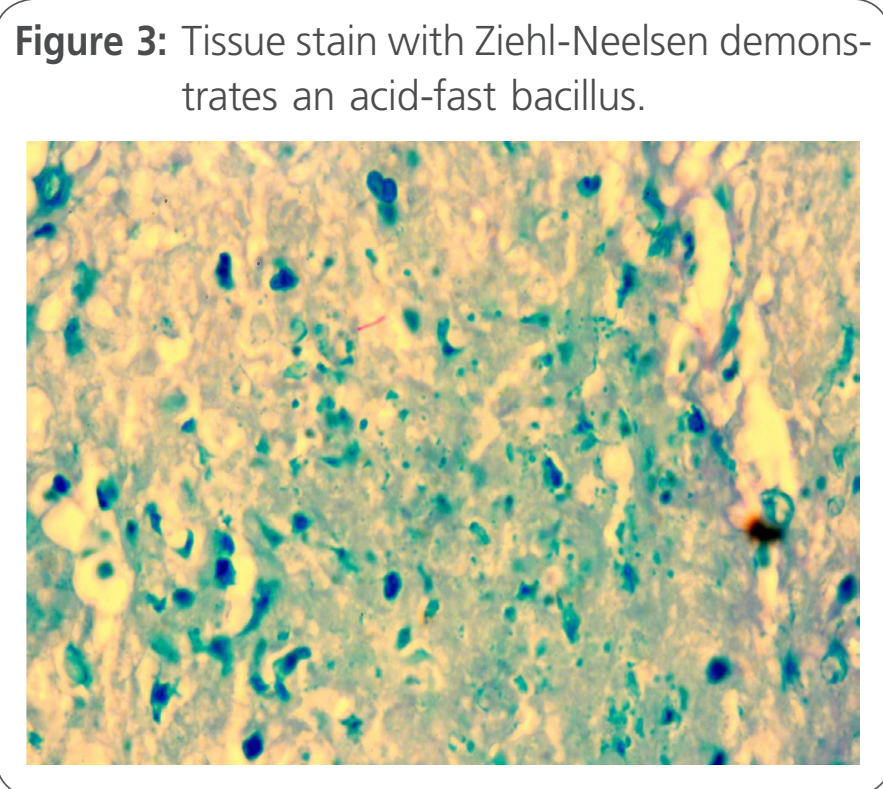

Figure 4: Acid fast bacilli grown in six weeks on Lowenstein-Jensen media and stained with Ziehl-Neelsen stain.

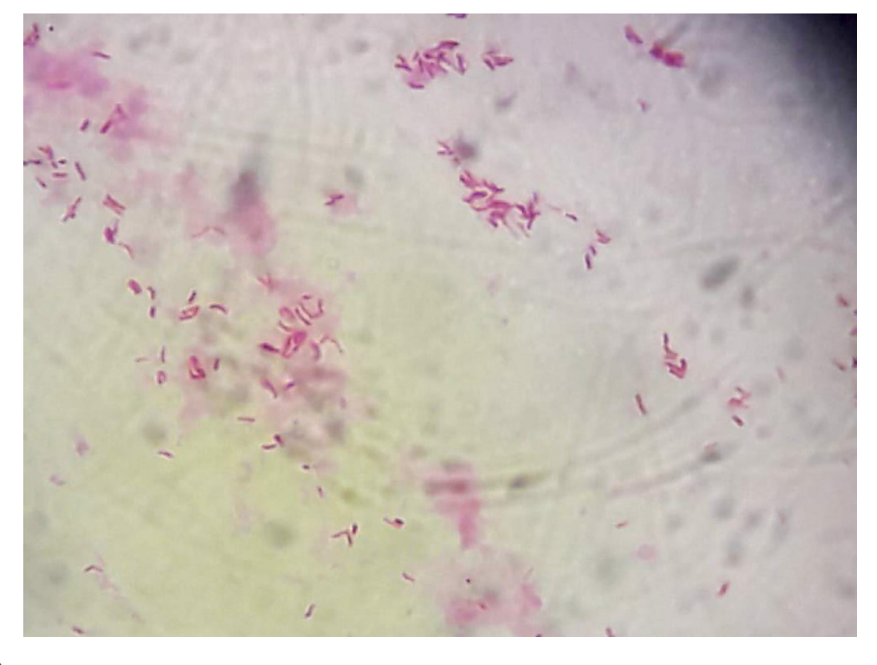

tive, described as "detected low". QuantiFERON-TB was negative. Pericardial fluid culture grew M. tuberculosis on Lowenstein-Jensen media in six weeks and was AFB stained (Figure 4). The patient was started on anti-tuberculosis treatment (isoniazid, rifampin, ethambutol and pyrazinamide) plus vitamin B6 with moxifloxacin in the first four weeks. He showed a progressive improvement, declining fever with normalizing sodium ( $\mathrm{Na} 133 \mathrm{mmol} / \mathrm{L}$ ), declining procalcitonin $(0.704 \mathrm{ng} / \mathrm{ml})$ and he was discharged 
from the hospital. As an outpatient, he was afebrile without serious complaints but mild pain at the site of his chest wound, though the surgical wound was clean and completely healed, also he complained from low back pain, and a new MRI spine was normal. His oral temperature was $36^{\circ} \mathrm{C}$, and other physical signs were unremarkable.

\section{Discussion}

Despite the patient was sick for one month and progressively getting worse, his initial presentation seemed like a bacterial sepsis, purulent bacterial pericarditis was suspected, and he was started on cefazolin [7]. CT scan of the heart showed pericardial effusions with signs like what was reported from 2D-echocardiography in Sudan of having unique signs (thickened effusion, fibrin strands, and shaggy pericardium) that support an early tuberculous pericarditis diagnosis [8]. Pericardial biopsy and later M. tuberculosis growth documented the diagnosis. Despite he was started on isoniazid, rifampin, ethambutol, and pyrazinamide plus B6, he showed improvement but fever continued few more days, moxifloxacin was added and fever resolved. An extensive review on the causes of pericarditis conducted by Noubiap JJ, et al. revealed that M. tuberculosisis a responsible agent in pericarditis, but not hemorrhagic pericarditis, and furthermore, no unique 2D-echocardiographic signs were detected [9]. A cross-sectional study from Sudan between January 2011 and June 2012 enrolled 985 patients, 22.7\% (224) were extrapulmonary tuberculosis, only $1.9 \%$ [4] patients were pericardial, and none were hemorrhagic [10]. Our patient diagnosis was firmly based on bacterial culture (Figure 4), and on pericardial biopsywith the characteristic picture (Figure $2 \mathrm{~A}, \mathrm{~B}$ ) and AFB on Ziehl-Neelsen stain of the pericardial biopsy (Figure 3). Notably, the QuantiFERON-TB test was negative, false negativeQuantiFERON-TB were reported in active tuberculosis infectionswith rates between $7.1 \%$ to $13.9 \%$ [11-12], and false negati- ve results may be source-dependent [13]. PCR was weakly positive, however, PCR methodology for the target genes may affect the results [14].

\section{Conclusion}

Our case is a rare presentation of Mycobacterium tuberculosis infection of the pericardium causing hemorrhagic pericarditis, and it was confirmed by histological and microbiological tests.

\section{References}

1. Azarbal A, LeWinter MM. Pericardial effusion. Cardiol Clin 2017; 35(4):515-524

2. Chang SA. Tuberculous and Infectious Pericarditis. Cardiol Clin 2017; 1; 35(4):615-22

3. Agarwal N, Chaturvedi A, Agarwal S, Mehra D, Kumar A, Statiction MS. Prevalence of tubercular pericardial effusion in Rohilkhand region, a prospective study. IJAR 2017; 3(9): 298301.

4. Atar S, Chiu J, Forrester JS, Siegel RJ. Bloody pericardial effusion in patients with cardiac tamponade: is the cause cancerous, tuberculous, or iatrogenic in the 1990s?. Chest 1999; 116(6): 1564-1569.

5. Lather HD, Kahlenberg JM. Hemorrhagic Pericardial Effusion with Tamponade: A Rare Adverse Effect of Infliximab-Case Report and Literature Review. Case Rep Rheumatol 2016; 2016.

6. Abugroun A, Hallak O, Ahmed F, Gaznabi S. Massive Hemorrhagic Pericardial Effusion With Cardiac Tamponade as Initial Manifestation of Mixed Connective Tissue Disease. Cardiol Res 2018; (1):68.

7. Modesto dos Santos V, dos Santos M, Arruda L. Hemopericardium, cardiac tamponade, and liver abscess in a young male. Neumol Cirugía de Tórax 2018; 76(4):325-8.

8. Alkhalifa MS, Elnima M, Ismaeel SA. Echocardiography in helping to determine the Causes of Pericardial Effusion in the Sudanese patients. Sudan J Med Sci 2009; 4(1).

9. Noubiap JJ, Agbor VN, Ndoadoumgue AL, Nkeck JR, Kamguia A, Nyaga UF, et al. Epidemiology of pericardial diseases in Africa: a systematic scoping review. Heart. 2019; 105(3):180-8.

10. Abdallah TE, Toum FE, Bashir OH, Mansoor TI, Yuosif MM, Elkhawad MA, et al. Epidemiology of extra pulmonary tuberculosis in Eastern Sudan. Asian Pac J Trop Biomed 2015; 5(6):505-8. 
11. Ak O, Dabak G, Ozer S, Saygi A, Dabak R. The evaluation of the Quantiferon-TB Gold test in pulmonary and extrapulmonary tuberculosis. Jpn J Infect Dis. 2009; 1; 62(2):149-51.

12. Kobashi Y, Shimizu H, Ohue Y, Mouri K, Obase Y, Miyashita N, et al. False negative results of QuantiFERON TB-2G test in patients with active tuberculosis. Jpn J Infect Dis 2009; 62(300):e2.

13. Kim YJ, Kang JY, Kim SI, Chang MS, Kim YR, Park YJ. Predictors for false-negative QuantiFERON-TB Gold assay results in patients with extrapulmonary tuberculosis. BMC Infect Dis 2018; 18(1):457

14. Kaur IR, Kashyap B, Goel N, Avasthi R, Vaid N, Arora V, et al. Utility of PCR targeting IS6110 and MPT64 genes in the diagnosis of extrapulmonary tuberculosis. Indian J Med Special 2017; 8(3):139-45.

Publish in The International Arabic Journal of Antimicrobial Agents

The Journal is an open access peer-reviewed journal that publishes scientific papers about all aspects of antimicrobials. The journal will publish original research articles, reviews, brief reports and case reports dealing with basic and clinical antibacterial agents, antiviral, antiprotozoals, antituberculuous, antifungal and antihelminthes agents. All manuscripts must be prepared in English, and are subject to a rigorous and fair peer-review process. Accepted papers will immediately appear online. The journal aims to advance the knowledge, attitude and the research of chemotherapy in the Arabic world in cooperation with international, national scientific and public societies as well as research centers with similar aims and objectives. 